\title{
Upregulation of miR-614 promotes proliferation and inhibits apoptosis in ovarian cancer by suppressing PPP2R2A expression
}

\author{
$\mathrm{JING}_{\mathrm{ZHANG}}{ }^{1^{*}}, \mathrm{DONGDONG} \mathrm{GAO}^{2^{*}}$ and HUI ZHANG ${ }^{1}$ \\ Departments of ${ }^{1}$ Traditional Chinese Medicine Gynecology and ${ }^{2}$ Oncology, \\ Central Hospital of Zhumadian, Huang Huai University, Zhumadian, Henan 463000, P.R. China
}

Received March 31, 2017; Accepted November 16, 2017

DOI: $10.3892 / \mathrm{mmr} .2018 .8714$

\begin{abstract}
It has previously been demonstrated that microRNAs (miRNAs) have essential roles and participate in various biological processes by regulating their specific target genes. However, the precise role of miRNAs in ovarian cancer (OC) has not yet been elucidated. The present study demonstrated that miR-614 expression levels were significantly upregulated in OC tissues and cell lines, whereas decreased miR-614 demonstrated opposite effects. Furthermore, gain-of-function and loss-of-function experiments indicated that miR-614 overexpression promoted cell proliferation and suppressed cell apoptosis. Protein phosphatase 2 regulatory subunit $\mathrm{B} \alpha$, (PPP2R2A) was identified as a direct target of miR-614 using western blotting and luciferase reporter assays. Notably, silencing of PPP2R2A counter-acted the effect of miR-614 inhibitor in OC cell proliferation and cell apoptosis. Overall, the data suggested that miR-614 promoted cell proliferation and inhibited cell apoptosis of OC cells by targeting PPP2R2A, and may therefore act as a potential target for OC therapy in the future.
\end{abstract}

\section{Introduction}

Ovarian cancer (OC) presents as the most fatal of the gynecological malignancies and is the leading cause of mortality among women worldwide (1). Although improvements and advancements have been made in current treatments of OC by combining surgery, chemotherapy, radiotherapy and immunotherapy, the survival rate of OC patients has not sufficiently improved (2). Therefore, it is necessary to identify novel

Correspondence to: Dr Hui Zhang, Department of Traditional Chinese Medicine Gynecology, Central Hospital of Zhumadian, Huang Huai University, 6 Kaiyuan Road, Zhumadian, Henan 463000, P.R. China

E-mail: hzhanghenan@126.com

${ }^{*}$ Contributed equally

Key words: microRNA-614, ovarian cancer, protein phosphatase 2 regulatory subunit $\mathrm{B} \alpha$, cell proliferation molecular biomarkers and therapeutic strategies for the treatment of patients suffering from OC.

MicroRNAs (miRNAs) are recognized as small, endogenous RNAs ( 23 nucleotides), which act as tumor suppressors or oncogenes and exhibit various important roles in carcinogenesis (3-6). It is reported that the dysregulation of miRNAs is frequently associated with cancer progression, including ovarian cancer (7-9). It is reported that miR-509-3p strongly attenuates multi-cellular spheroids and cell metastasis of ovarian cancer (10). Zhang et al (11) suggests that miR-520 g represses death associated protein kinase 2 and then promotes epithelial OC progression and chemo-resistance. Wu et al (12) noted that miR-572 promotes cell proliferation of human ovarian cancer cells by repressing protein phosphatase 2 regulatory subunit B $\alpha$ (PPP2R2A) expression. miR-381 is reported to suppress cell proliferation, migration and invasion of epithelial OC via suppression of YY1 transcription factor expression (13). However, the biological roles and underlying mechanisms of miR-614 during the pathogenesis of OC have not yet been clearly elucidated. In the present study, it was demonstrated that miR-614 was upregulated in OC clinical tissues and cell lines. Ectopic overexpression of miR-614 promoted the cell proliferation and colony-forming abilities, and decreased the apoptotic rate of A2780 cells in vitro. Furthermore, it was identified that PPP2R2A is a direct target of miR-614. Overall, the findings indicated that miR-614 regulated OC cell proliferation, colony formation abilities and cell apoptosis, by directly modulating PPP2R2A.

\section{Materials and methods}

Tissue samples and cell culture. Written informed consent was obtained from all patients (age range, 32-55) at the Department of Traditional Chinese Medicine Gynecology, Huang Huai University (Zhumadian, China) that participated in the study, and the study was approved by the Ethics Committee of Huang Huai University. None of the patients had received chemoradiotherapy. A total of 8 pairs of primary ovarian carcinoma tissues ( $\mathrm{T}$ ) and the matched adjacent non-tumor normal tissues (ANT) were collected from patients having undergone surgery. A total of two fresh normal ovarian tissues were each collected from two patients. All specimens had been verified with pathological diagnosis. Tissue samples were frozen and stored in liquid nitrogen until their use in western blotting and 
reverse transcription-quantitative polymerase chain reaction (RT-qPCR).

Human ovarian cancer cell lines COV362, SKOV3, EFO27, COV644, A2780, OV90, CAOV3 and IGROV-1 were purchased from the Shanghai Institute of Biochemistry and Cell Biology, Chinese Academy of Sciences (Shanghai, China). Human normal ovarian epithelial cell line IOSE80 and ovary surface epithelial cells HOSE were all purchased from Shanghai Huiying Biological Technology Co., Ltd. (Shanghai, China). Cells were cultured in Dulbecco's modified Eagle's medium (Gibco; Thermo Fisher Scientific, Inc., Waltham, MA, USA) supplemented with $10 \%$ fetal bovine serum (Gibco; Thermo Fisher Scientific, Inc.). All cell lines were maintained at $37^{\circ} \mathrm{C}$ in a humidified incubator in an environment containing $5 \% \mathrm{CO}_{2}$.

miRNAs, small interfering (si)RNA and transfection. The miR-614 mimic (HmiR0183-MR04; GeneCopoeia, Inc., Rockville, MD, USA), miR-614 inhibitor (miR-614-in; HmiR-AN0718-AM02; GeneCopoeia, Inc.), the corresponding negative controls (Vector and NC) and siRNA-PPP2R2A (PPP2R2A-siRNA\#1, HSH014174; PPP2R2A-siRNA\#2 and HSH055016) were purchased from GeneCopoeia, Inc., and A2780 cells (Shanghai Institute of Biochemistry and Cell Biology, Chinese Academy of Sciences, Shanghai, China) were plated and cultured overnight and transfected with $50 \mathrm{nM}$ indicated RNA using Lipofectamine ${ }^{\circledR} 2000$ (Invitrogen; Thermo Fisher Scientific, Inc.) following the manufacturer's protocol. Cells were harvested 24 or $48 \mathrm{~h}$ after transfection.

RNA extraction and RT- $q P C R$. Total RNA was extracted from clinical tissues and cancer cells using TRIzol ${ }^{\circledR}$ reagent (Thermo Fisher Scientific, Inc.) and reverse transcribed to cDNA using a Reverse Transcription kit (Takara Biotechnology Co., Ltd., Dalian, China) according to the manufacturer's protocol. The expression levels of miR-614, B cell lymphoma-2 associated agonist of cell death (BAD) and Cyclin D1 were measured by RT-qPCR with SYBR-Green Mix Taq kit (Takara Bio, Inc., Otsu, Shiga, Japan) in an ABI Prism7500 Sequence Detection System (Applied Biosystems; Thermo Fisher Scientific, Inc.). The following PCR primers were synthesized by GeneCopoeia, Inc.: miR-614 (HmiRQP0718), BAD (HQP015538) and Cyclin D1 (HQP016204). U6 (HmiRQP9001) and GAPDH (HQP064347) were used as internal controls for normalization. Relative expression levels were calculated using the $2^{-\Delta \Delta \mathrm{Cq}}$ method (14).

MTT and 5-bromodeoxyuridine (BrdU) incorporation assays. Cell proliferation was assessed using an MTT assay. Following transfection, A2780 cells were tested using the MTT assay at different time points. A total of $20 \mu \mathrm{l}$ of $5 \mathrm{mg} / \mathrm{ml}$ MTT solution (Sigma-Aldrich; Merck KGaA, Darmstadt, Germany) was added to each well and $150 \mu \mathrm{l}$ dimethyl sulfoxide was added then incubated for $4 \mathrm{~h}$ at $37^{\circ} \mathrm{C}$. Following this, the absorbance was measured using a microplate reader at a wavelength of $490 \mathrm{~nm}$.

Cell growth was measured using a BrdU incorporation assay (EMD Millipore, Billerica, MA, USA). Following transfection and culturing for $24 \mathrm{~h}, \mathrm{~A} 2780$ were incubated with BrdU for $1 \mathrm{~h}$, and stained with BrdU antibodies (cat. no. 61273; 1:500; Upstate Biotechnology, Inc., Lake Placid,
$\mathrm{NY}$, USA) at $4^{\circ} \mathrm{C}$ overnight, and then incubated with horseradish peroxidase-modified secondary antibodies (ab6741; 1:5,000; Abcam, Cambridge, UK) at $37^{\circ} \mathrm{C}$ for $2 \mathrm{~h}$. Gray level images were measured under a laser-scanning microscope (Axioskop 2 plus; Carl Zeiss Co., Ltd., Jena, Germany).

Anchorage-independent growth ability assay. For the anchorage-independent growth ability assay, a 1.5-ml basal layer of $0.8 \%$ agar (Sigma-Aldrich; Merck KGaA) was poured into a 6-well culture plate. A total of 500 cells (quantified using a Z2 Coulter Counter Analyzer; Beckman Coulter, Inc., Brea, CA, USA) were trypsinized and suspended in $2 \mathrm{ml}$ complete medium plus $0.3 \%$ agar and then added to each well, and incubated at $37^{\circ} \mathrm{C}$ in an environment containing $5 \% \mathrm{CO}_{2}$, in a humidified incubator for 2 weeks. Colonies greater than $0.1 \mathrm{~mm}$ in diameter were counted manually.

Cell apoptosis assay. Following transfection A2780 cells $\left(2 \times 10^{5}\right)$ were plated into 6-well plates. After $48 \mathrm{~h}$, cells were digested with $0.25 \%$ trypsin, centrifuged, and the supernatants discarded. The cell pellets were washed three times with cold PBS and the supernatants discarded. The cells were then incubated with $5 \mu 1$ Annexin V-FITC (C1062; Beyotime Institute of Biotechnology, Haimen, China) and $5 \mu 1$ propidium iodide (PI; C1062; Beyotime Institute of Biotechnology) for $15-20 \mathrm{~min}$ in the dark at $25^{\circ} \mathrm{C}$, and then the percentage of apoptotic cells was detected by a BD FACSVerse flow cytometer (BD Biosciences, Franklin Lakes, NJ, USA) and analyzed using the BD FACStation data management system.

Bioinformatics analysis. To investigate the target gene of miR-614, TargetScan version 7.1 (http://www.targetscan.org) was used to predict the potential target gene of miR-614.

Luciferase assay. The wild type 3'-untranslated region (UTR) PPP2R2A vectors were co-transfected with miR-614 or miR-614-mutated or relative negative controls, into A2780 cells using Lipofectamine ${ }^{\mathrm{TM}} 2000$ reagent (Invitrogen; Thermo Fisher Scientific, Inc.), following the manufacturer's protocol. Following $48 \mathrm{~h}$, cells were lysed and the Luciferase reporter assays were measured using a Dual-Luciferase Reporter Assay System (Promega, Madison, WI, USA). The luciferase activity was normalized to Renilla luciferase activity.

Western blotting. Protein samples were lysed from OC cells that underwent transfection, using a radioimmunoprecipitation assay lysis buffer (Cell Signaling Technology, Inc., Danvers, MA, USA). Protein samples were quantified with the Pierce BCA Protein assay kit (Pierce; Thermo Fisher Scientific, Inc.) and were then boiled for $10 \mathrm{~min}$ in sodium dodecyl sulfate (SDS) sample buffer. Protein samples $(20 \mu \mathrm{g})$ were separated with $10 \%$ SDS-PAGE, and subsequently transferred onto polyvinylidene membranes via electroblotting. Following blocking with $5 \%$ dried milk for $2 \mathrm{~h}$ at room temperature, membranes were then incubated with anti-PPP2R2A, anti-Cyclin D1 (cat. no. 2978; 1:1,000), anti-BAD, anti-phosphorylated (p)-Rb (cat. no. 8516; 1:1,000), anti-Rb (cat. no. 9313; 1:1,000) and anti- $\alpha$-tubulin antibodies (cat. no. 2144; 1:1,000; all from Cell Signaling Technology, Inc.) overnight at $4^{\circ} \mathrm{C}$, and then incubated with corresponding horseradish peroxidase-conjugated 
A

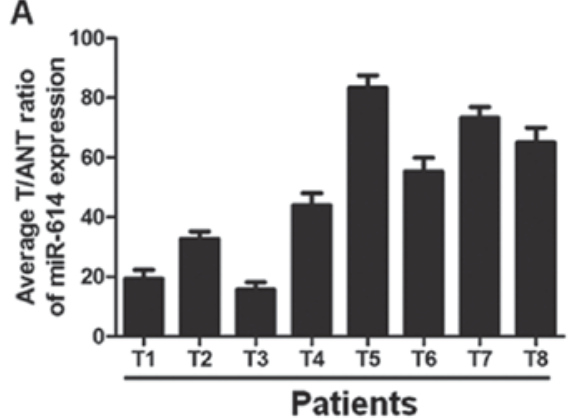

B

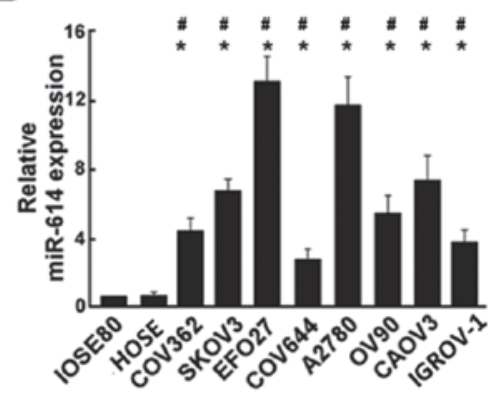

Figure 1. Expression of miR-614 in human OC tissues and cell lines. (A) Relative miR-614 mRNA expression levels in 8 paired primary OC tissues (T) and the matched adjacent non-tumor normal tissues (ANT) from the same patient were detected by RT-qPCR analysis. (B) RT-qPCR analysis of miR-614 expression in human normal ovarian epithelial cell line IOSE80, ovary surface epithelial cells HOSE and OC cell lines, including COV362, SKOV3, EFO27, COV644, A2780, OV90, CAOV3 and IGROV-1. "P<0.05 vs. IOSE80; ${ }^{\mathrm{P}}<0.05$ vs. HOSE. miRNA, microRNA; OC, ovarian cancer; RT-qPCR, reverse transcription-quantitative polymerase chain reaction.

A

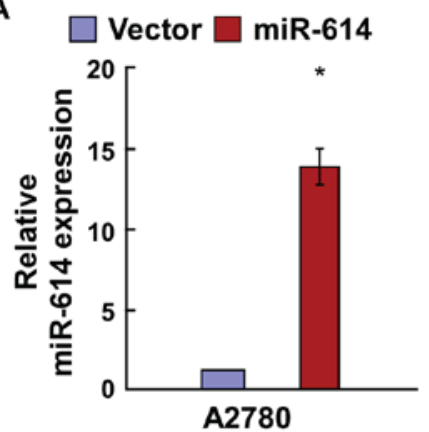

C

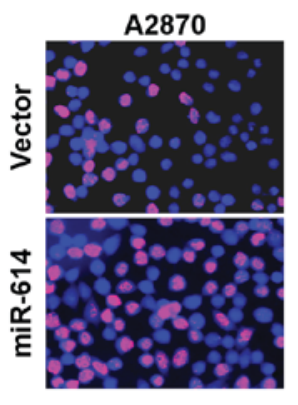

E

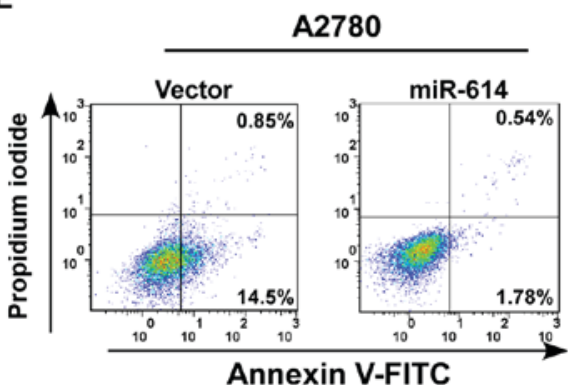

B

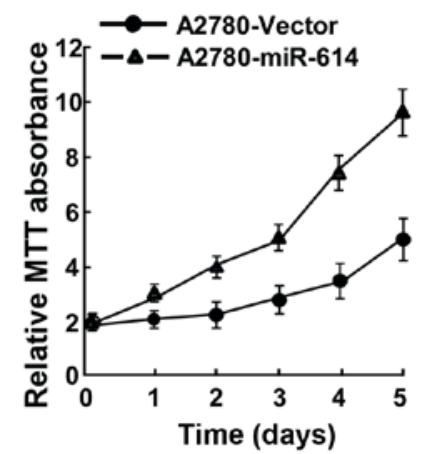

D
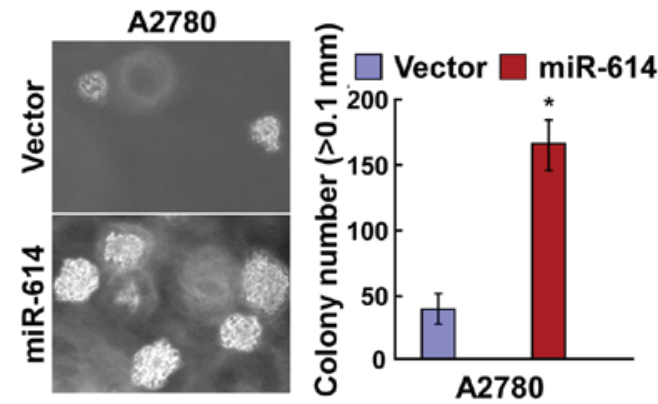

Figure 2. Upregulation of miR-614 promotes ovarian cancer cell proliferation and decreases cell apoptosis. (A) Validation of miR-614 expression levels following transfection, detected by reverse transcription-quantitative polymerase chain reaction. (B) MTT assays revealed that upregulation of miR-614 promoted growth of A2780 cells. (C) Representative micrographs (left) and quantification (right) of the BrdU incorporation assay in A2780 cells. (D) Upregulation of miR-614 promoted the anchorage-independent growth of A2780 cells. Representative micrographs (left) and quantification of colonies that were $>0.1 \mathrm{~mm}$ (right). (E) Cell apoptosis rate was decreased in miR-614 mimics group. Each bar represents the mean of three independent experiments. ${ }^{*} \mathrm{P}<0.05$ vs. Control. miRNA, microRNA; BrdU, 5-bromodeoxyuridine.

secondary antibodies (cat. no. 7074; 1:5,000, Cell Signaling Technology, Inc.) for $2 \mathrm{~h}$ at room temperature. The protein bands were detection by an enhanced chemiluminescence western blotting substrate (Pierce; Thermo Fisher Scientific, 
A

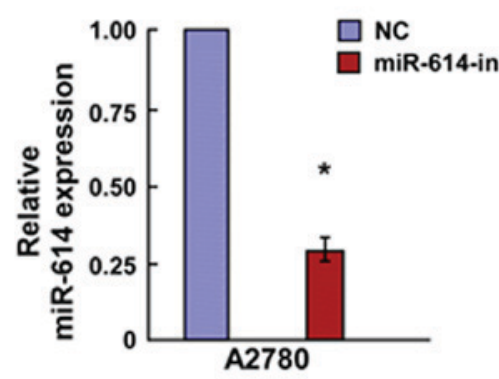

C
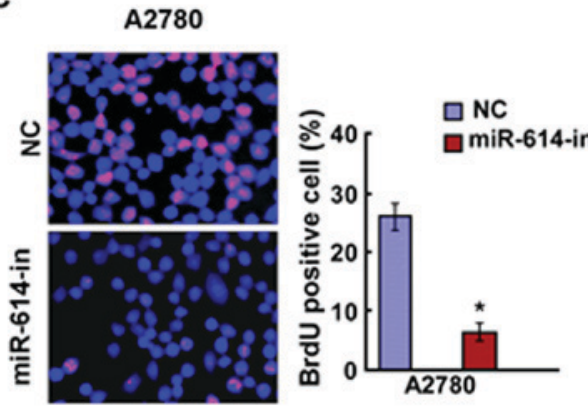

E

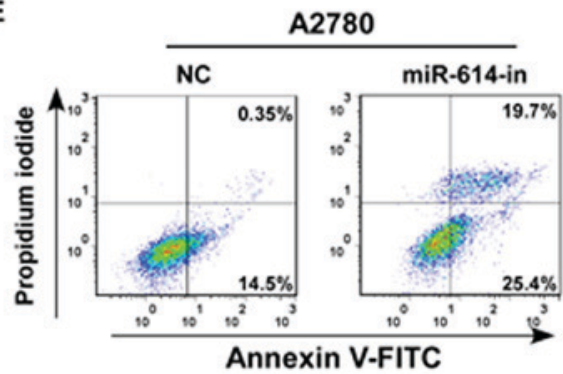

B

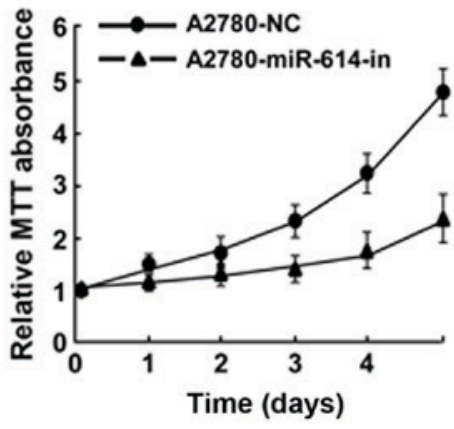

D
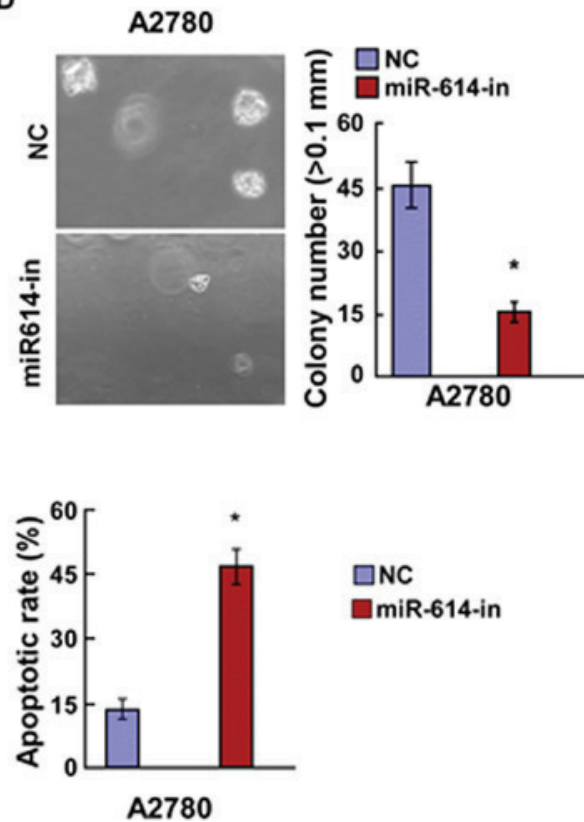

$\square N C$

DmiR-614-in

Figure 3. Inhibition of miR-614 inhibited ovarian cancer cell proliferation and increased cell apoptosis. (A) Validation of miR-614 expression levels following transfection, using reverse transcription-quantitative polymerase chain reaction. (B) MTT assays revealed that miR-614-in suppressed growth of A2780 cells. (C) Representative micrographs (left) and quantification (right) of the BrdU incorporation assay in A2780 cells. (D) miR-614-in inhibited the anchorage-independent growth of A2780 cells. Representative micrographs (left) and quantification of colonies that were $>0.1 \mathrm{~mm}$ (right). (E) Cell apoptosis rate was increased in miR-614-in group. Each bar represents the mean of three independent experiments. * $\mathrm{P}<0.05$ vs. Control. miRNA, microRNA; in, inhibitor; BrdU, 5-bromodeoxyuridine; NC, negative control.

Inc.) and quantified using ImageJ software version 1.48 (National Institutes of Health, Bethesda, MD, USA).

Statistical analysis. All statistical analyses were presented as the mean \pm standard deviation and were conducted using SPSS software, version 19.0 (IBM SPSS, Armonk, NY, USA). Comparisons between groups were evaluated by a Student's t-test or one-way analysis of variance followed by a post hoc Tukey test for multiple comparisons. $\mathrm{P}<0.05$ was considered to indicate a statistically significant difference.

\section{Results}

miR-614 expression is upregulated in OC tissues and cell lines. RT-qPCR analysis was firstly performed to examine the expression of miR-614 in primary ovarian carcinoma tissues (T) and the matched adjacent non-tumor normal tissues (ANT), and the results revealed that miR-614 levels in primary ovarian carcinoma tissues were significantly increased compared with adjacent non-tumor normal tissues (Fig. 1A). miR-614 expression levels were then further detected in eight ovarian cancer cell lines (COV362, SKOV3, EFO27, COV644, A2780, OV90, CAOV3 and IGROV1), human normal ovarian epithelial cell line IOSE80 and the ovary surface epithelial cells HOSE, and the data indicated that miR-614 was markedly upregulated in ovarian cancer cell lines (Fig. 1B), providing evidence that miR-614 may be associated with ovarian cancer progression.

miR-614 promotes cell proliferation of $O C$. Due to the upregulated expression of miR-614 in OC clinical tissues and cell lines, the present study next investigated its effects on OC cell lines. miR-614 mimic and the respective controls were transfected into A2780 OC cells, and then the transfection efficiency was verified by RT-qPCR (Fig. 2A). Results of the MTT and BrdU assays revealed that miR-614 overexpression significantly increased cell proliferation of A2780 cells (Fig. 2B and C), and this was further verified by the anchorage-independent growth ability assay (Fig. 2D). Cell apoptosis analysis indicated that the apoptotic rate was significantly decreased in A2780 cells following transfection with miR-614 in comparison with the controls 


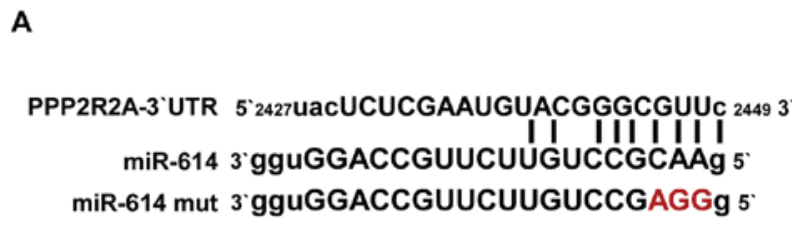

C
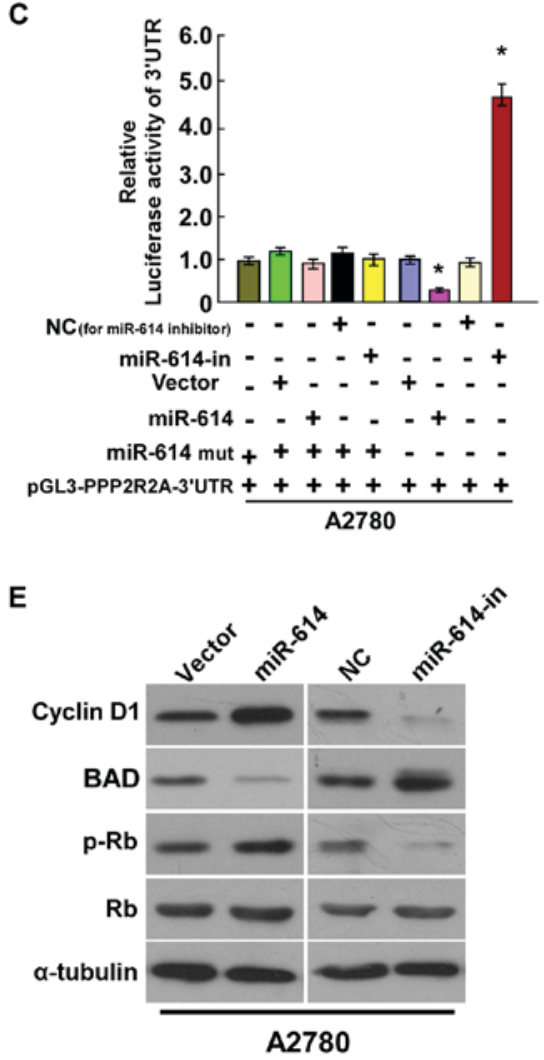

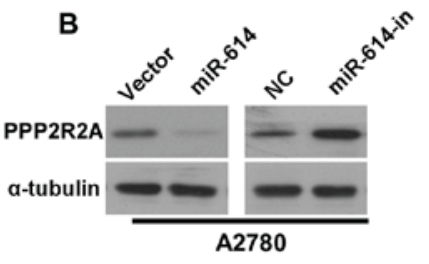

D

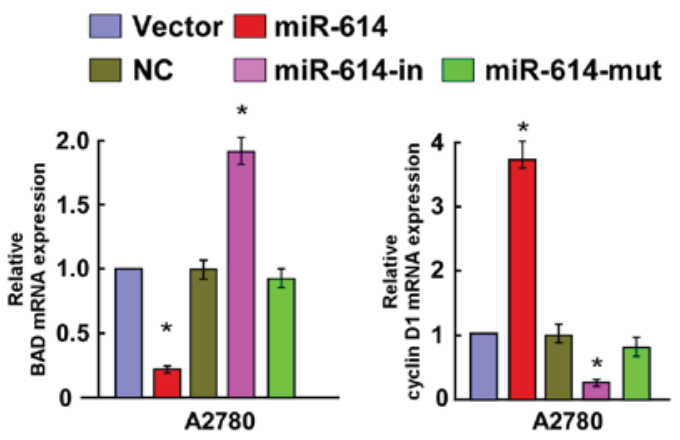

Figure 4. miR-614 suppresses PPP2R2A expression by directly targeting the PPP2R2A 3'-UTR and altered levels of proteins associated with cell proliferation and cell apoptosis in A2780 cells. (A) Predicted miR-614c target sequence in the 3'-UTR of PPP2R2A (PPP2R2A-3'-UTR) and positions of three mutated nucleotides (red) in the 3'-UTR of miR-614 (miR-614-mut). (B) Expression of PPP2R2A in A2780 cells transfected with miR-614 or the miR-614-in were detected by western blotting analysis. $\alpha$-tubulin served as the loading control. (C) Luciferase reporter assay of A2780 cells transfected with the pGL3-PPP2R2A-3'-UTR reporter and miR-614 or miR-614-in or miR-614-mut oligonucleotides. (D) Reverse transcription-quantitative polymerase chain reaction analysis of expression of BAD and Cyclin D1 in A2780 cells. (E) Western blot analysis of protein expression of Cyclin D1, BAD, p-Rb and Rb in A2780 cells. $\alpha$-tubulin served as the loading control. "P<0.05 vs. Control. BAD, B cell lymphoma-2 associated agonist of cell death; miRNA, microRNA; in, inhibitor; UTR, untranslated region; PPP2R2A, protein phosphatase 2 regulatory subunit $\mathrm{B} \alpha$; NC, negative control; p, phosphorylated; mut, mutated.

(Fig. 2E). miR-614 inhibitor and the respective controls were transfected into A2780 OC cells, and the transfection efficiency was verified by RT-qPCR (Fig. 3A). Results of the MTT and BrdU assays indicated that miR-614-in significantly decreased cell proliferation of A2780 cells (Fig. 3B and C), and this was further verified by the anchorage-independent growth ability assay (Fig. 3D). Cell apoptosis analysis revealed that miR-614-in promoted cell apoptosis rate in A2780 cells (Fig. 3E). Overall, miR-614 is capable of promoting cell proliferation of OC which is associated with the regulation of cell apoptosis.

miR-614 directly targets PPP2R2A by binding to its 3'-UTR, and alters levels of proteins associated with proliferation in $O C$. To explore the regulatory mechanism of miR-614 in $\mathrm{OC}$ cell proliferation, the present study used algorithm programs (Target Scan software; http://www.targetscan.org/vert_50/) and identified that PPP2R2A 3'-UTR contained a predicted binding site of miR-614 (Fig. 4A). The western blotting assay revealed that miR-614 overexpression suppressed PPP2R2A expression, whereas miR-614-in increased its expression (Fig. 4B). The Luciferase activity assay indicated that co-transfection of miR-614 and pGL3-PPP2R2A 3'-UTR resulted in a significant reduction in luciferase activity, whereas the luciferase activity was significantly increased with co-transfection of miR-614-in and pGL3- PPP2R2A 3'-UTR. However, there was no alteration in luciferase activity when co-transfection with miR-614-mut and the luciferase reporter vector occurred. Together, the results suggested that miR-614 directly binds to the 3'UTR of PPP2R2A (Fig. 4C). Given that the results revealed that miR-614 regulated cell proliferation and cell apoptosis of OC, the effects on the expression level of PPP2R2A downstream genes were then investigated, including Cyclin D1, BAD, Rb and $\mathrm{p}-\mathrm{Rb}$. As the results of the RT-qPCR indicated, miR-614 significantly decreased the mRNA expression level of the apoptotic gene BAD and increased expression of the cell proliferation associated gene Cyclin D1, whereas miR-614-in revealed the opposite effect, and miR-614-mut had no effect on their mRNA expression levels (Fig. 4D). Western blot assays revealed that 
A

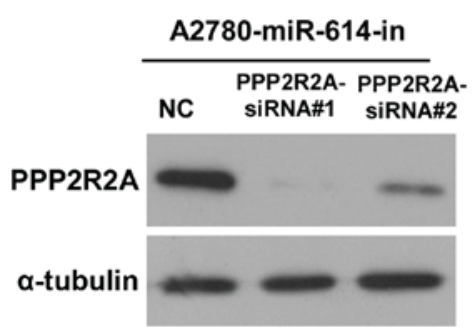

C

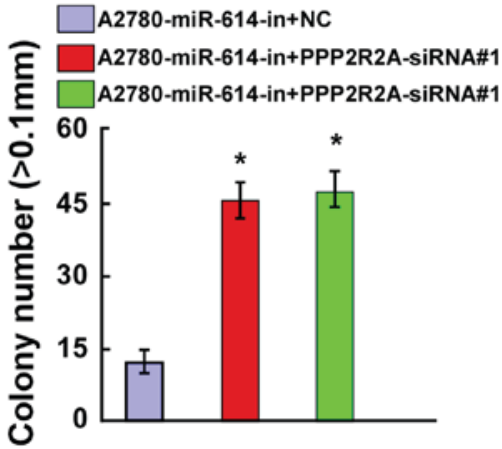

B

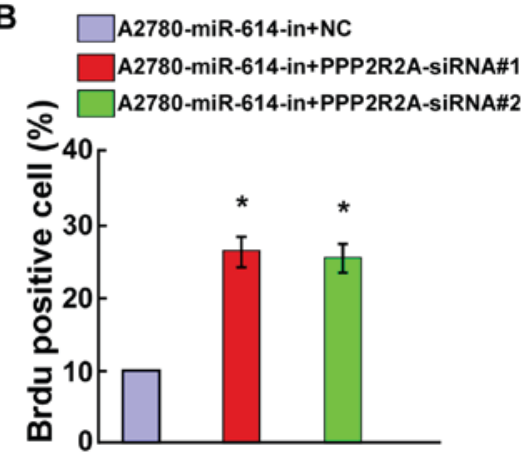

D

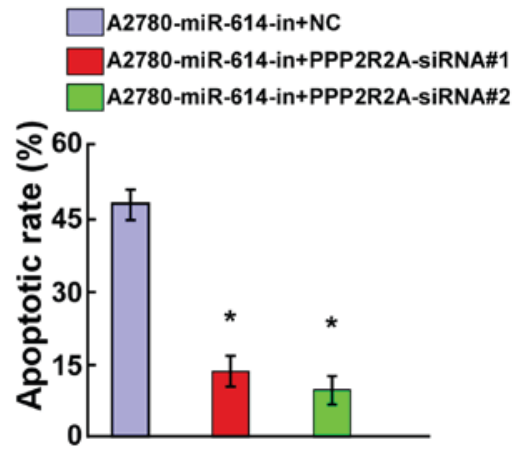

Figure 5. Knockdown of PPP2R2A counteracted effect of miR-614-in on ovarian cancer cell proliferation and cell apoptosis. (A) Western blot analysis verified that silencing PPP2R2A effectively decreased the expression of PPP2R2A in miR-614-in-transfected A2780 cells. (B) BrdU incorporation assay was used to detect miR-614-in-transfected A2780 cells following transfection with PPP2R2A-siRNAs. (C) miR-614-in-transfected A2780 cells following transfection with PPP2R2A-siRNAs promoted the anchorage-independent growth. Representative quantification of colonies that were $>0.1 \mathrm{~mm}$. (D) Cell apoptosis rate was decreased in miR-614-in-transfected A2780 cells following transfection with PPP2R2A-siRNAs. Each bar represents the mean of three independent experiments. " $\mathrm{P}<0.05$ vs. Control. miRNA, microRNA; in, inhibitor; PPP2R2A, protein phosphatase 2 regulatory subunit B $\alpha$ II; NC, negative control; si, small interfering; BrdU, 5-bromodeoxyuridine.

overexpression of miR-614 enhanced the protein expression levels of Cyclin D1 and $\mathrm{p}-\mathrm{Rb}$ and decreased the BAD protein expression, whereas miR-614-in significantly reduced the protein expression levels of Cyclin D1 and $\mathrm{p}-\mathrm{Rb}$ and increased the BAD protein expression (Fig. 4E). Collectively, these results demonstrated that miR-614 regulated OC cell growth and cell apoptosis by targeting PPP2R2A.

Knockdown of PPP2R2A counteracted the effect of miR-614-in on $O C$ cell proliferation and cell apoptosis. To verify the role of PPP2R2A in OC cell proliferation, it was investigated whether the knockdown of PPP2R2A could counteract the proliferation arrest induced by miR-614-in. The siRNA interference efficiency of PPP2R2A-siRNAs was evaluated by western blot analysis (Fig. 5A). The BrdU positive rate of miR-614-in transfected A2780 cells was increased following transfection with PPP2R2A-siRNAs (Fig. 5B). Additionally, the anchorage-independent growth ability assay demonstrated that the anchorage-dependent proliferation induced by miR-614-in could be abolished by knockdown of PPP2R2A (Fig. 5C). Furthermore, the results of the cell apoptosis assay indicated that miR-614-in increased the apoptotic rate, which was then attenuated by knockdown of PPP2R2A in A2780 cells (Fig. 5D).

\section{Discussion}

The present study, to the best of the author's knowledge, demonstrated for the first time, that miR-614 was upregulated in OC clinical tissues and cells. Increased expression of miR-614 led to the promotion of cell proliferation and colony-forming abilities, and conversely, decreased the apoptotic rate of OC cells. Additionally, PPP2R2A may act as a novel target of miR-614. The present study indicated that miR-614 may act as a novel tumor promoter in OC by targeting PPP2R2A.

Accumulating evidence suggests that miRNAs exhibit an essential role in human cancer pathological proceedings via controlling different target genes, including those involved in cell proliferation, migration, invasion, cycle and apoptosis (15-18). Dysregulation of miRNA frequently occurs in novel types of cancers, including ovarian cancer. miR-21-3p inhibits cell proliferation and invasion of ovarian cancer by targeting RNA binding protein with multiple splicing, $\mathrm{RCC} 1$ and $\mathrm{BTB}$ domain containing protein 1 and Zinc finger protein 608 (19). Fu et al suggests that miR-222-3p suppresses epithelial OC cell growth by regulating $G$ protein subunit $\alpha$ I2 (17). miR-203 promotes cell growth and migration of $\mathrm{OC}$ by targeting pyruvate dehydrogenase (Lipoamide) $\beta$ (20). However, the functions of miR-614 in OC have not been fully elucidated. The data of the present study indicated that miR-614 expression was upregulated in OC clinical tissues and cell lines. Overexpression of miR-614 enhanced OC cell proliferation and decreased cell apoptosis rate, suggesting miR-614 exhibits an important role in OC progression.

PPP2R2A, a regulatory subunit of phosphatase, acts as a well-recognized regulator in the control of the AKT serine/threonine kinase signaling pathway associated with tumor growth (21-23). miR-136 promotes cell proliferation of human 
non-small cell lung cancer cells by targeting PPP2R2A (24). miR-31 acts as an oncogenic microRNA in human lung cancer cells by repressing PPP2R2A (25). Liang et al (26) indicates that miR-892a promotes cell proliferation of human colorectal cancer cells by regulating PPP2R2A expression. Wong et al (27) reports that miR-222 is overexpressed in hepatocellular carcinoma and promotes cell motility by targeting PPP2R2A. The present study identified that PPP2R2A was a potential target of miR-614 by using a bioinformatics search. Subsequently, western blotting and luciferase reporter assays demonstrated that miR-614 targeted PPP2R2A and suppressed its expression. Further experiments to investigate the mechanism underlying the PPP2R2C mediated cancer cell proliferation and cell apoptosis are required. Results from RT-qPCR and western blotting analysis indicated that BAD (mRNA and protein) levels were downregulated whereas Cyclin D1 (mRNA and protein) levels were upregulated in miR-614-transfected A2780 cells, whereas miR-614-in demonstrated the opposite effect. Furthermore, knockdown of PPP2R2A counteracted the effect of miR-614-in on OC cell proliferation and cell apoptosis.

In conclusion, the findings suggested that miR-614 expression was upregulated in OC clinical tissues and cells. Overexpression of miR-614 promoted cell proliferation and regulated cell apoptosis through inhibition of PPP2R2A. The findings suggested that miR-614 may act as a potential therapeutic target for the treatment of $\mathrm{OC}$ in the future.

\section{Acknowledgements}

Not applicable.

\section{Funding}

No funding was received.

\section{Availability of data and materials}

The datasets used and/or analyzed during the current study are available from the corresponding author on reasonable request.

\section{Authors' contributions}

JZ and DG designed and performed this study. HZ analyzed the data. All authors read and approved the final manuscript.

\section{Ethics approval and consent to participate}

Written informed consent was obtained from all patients (age range, 32-55) at the Department of Traditional Chinese Medicine Gynecology, Huang Huai University (Zhumadian, China) that participated in the study, and the study was approved by the Ethics Committee of Huang Huai University.

\section{Consent for publication}

Written informed consent was obtained from all patients.

\section{Competing interests}

The authors declare that they have no competing interests.

\section{References}

1. Siegel R, Ma J, Zou Z and Jemal A: Cancer statistics, 2014. CA Cancer J Clin 64: 9-29, 2014.

2. Krishnan V, Berek JS and Dorigo O: Immunotherapy in ovarian cancer. Curr Probl Cancer 41: 48-63, 2016.

3. Xie F, Yuan Y, Xie L, Ran P, Xiang X, Huang Q, Qi G, Guo X Xiao $\mathrm{C}$ and Zheng S: miRNA-320a inhibits tumor proliferation and invasion by targeting c-Myc in human hepatocellular carcinoma. Onco Targets Ther 10: 885-894, 2017.

4. Hu Y, Xie H, Liu Y, Liu W, Liu M and Tang H: miR-484 suppresses proliferation and epithelial-mesenchymal transition by targeting ZEB1 and SMAD2 in cervical cancer cells. Cancer Cell Int 17: 36, 2017

5. Gao Y, Lin L, Li T, Yang J and Wei Y: The role of miRNA-223 in cancer: Function, diagnosis and therapy. Gene 616: 1-17, 2017.

6. Arechaga-Ocampo E,Lopez-Camarillo C, Villegas-Sepulveda N, Gonzalez-De la Rosa CH, Perez-Añorve IX, Roldan-Perez R, Flores-Perez A, Peña-Curiel O, Angeles-Zaragoza O, Rangel Corona $\mathrm{R}$, et al: Tumor suppressor miR-29c regulates radioresistance in lung cancer cells. Tumour Biol 39: 1010428317695010, 2017.

7. Wang W, Yang J, Xiang YY and Pi J: Overexpression of Hsa-miR-320 Is associated with invasion and metastasis of ovarian cancer. J Cell Biochem 118: 3654-3661, 2017.

8. Xu J, Jiang N, Shi H, Zhao S, Yao S and Shen H: miR-28-5p promotes the development and progression of ovarian cancer through inhibition of N4BP1. Int J Oncol: Mar 16, 2017 (Epub ahead of print).

9. Bai L, Wang H, Wang AH, Zhang LY and Bai J: MicroRNA-532 and microRNA-3064 inhibit cell proliferation and invasion by acting as direct regulators of human telomerase reverse transcriptase in ovarian cancer. PLoS One 12: e0173912, 2017.

10. Pan Y, Robertson G, Pedersen L, Lim E, Hernandez-Herrera A, Rowat AC, Patil SL, Chan CK, Wen Y,Zhang X, et al: miR-509-3p is clinically significant and strongly attenuates cellular migration and multi-cellular spheroids in ovarian cancer. Oncotarget 7: 25930-25948, 2016.

11. Zhang J, Liu L, Sun Y, Xiang J, Zhou D, Wang L, Xu H, Yang X, Du N, Zhang M, et al: MicroRNA-520 g promotes epithelial ovarian cancer progression and chemoresistance via DAPK2 repression. Oncotarget 7: 26516-26534, 2016.

12. Wu AH, Huang YL, Zhang LZ, Tian G, Liao QZ and Chen SL: MiR-572 prompted cell proliferation of human ovarian cancer cells by suppressing PPP2R2C expression. Biomed Pharmacother 77: 92-97, 2016

13. Xia B, Li H, Yang S, Liu T and Lou G: MiR-381 inhibits epithelial ovarian cancer malignancy via YY1 suppression. Tumour Biol 37: 9157-9167, 2016.

14. Livak KJ and Schmittgen TD: Analysis of relative gene expression data using real-time quantitative PCR and the 2(-Delta Delta C(T)) method. Methods 25: 402-408, 2001.

15. Cao C, Sun D, Zhang L and Song L: miR-186 affects the proliferation, invasion and migration of human gastric cancer by inhibition of Twist1. Oncotarget 7: 79956-79963, 2016.

16. Lei ST, Shen F, Chen JW, Feng JH, Cai WS, Shen L, Hu ZW and Xu B: MiR-639 promoted cell proliferation and cell cycle in human thyroid cancer by suppressing CDKN1A expression. Biomed Pharmacother 84: 1834-1840, 2016.

17. Fu X, Li Y, Alvero A, Li J, Wu Q, Xiao Q, Peng Y, Hu Y, Li X, Yan W, et al: MicroRNA-222-3p/GNAI2/AKT axis inhibits epithelial ovarian cancer cell growth and associates with good overall survival. Oncotarget 7: 80633-80654, 2016.

18. Wen Z, Zhao S, Liu S, Liu Y, Li X and Li S: MicroRNA-148a inhibits migration and invasion of ovarian cancer cells via targeting sphingosine-1-phosphate receptor 1. Mol Med Rep 12: 3775-3780, 2015 .

19. Baez-Vega PM, Echevarria Vargas IM, Valiyeva F, EncarnaciónRosado J, Roman A, Flores J, Marcos-Martínez MJ and VivasMejía PE: Targeting miR-21-3p inhibits proliferation and invasion of ovarian cancer cells. Oncotarget 7: 36321-36337, 2016.

20. Xiaohong Z, Lichun F, Na X, Kejian Z, Xiaolan X and Shaosheng W: MiR-203 promotes the growth and migration of ovarian cancer cells by enhancing glycolytic pathway. Tumour Biol 37: 14989-14997, 2016. Zeng LP, Hu ZM, Li K and Xia K: miR-222 attenuates cisplatin-induced cell death by targeting the PPP2R2A/Akt/mTOR Axis in bladder cancer cells. J Cell Mol Med 20: 559-567, 2016. 
21. Zeng LP, Hu ZM, Li K and Xia K: miR-222 attenuates cisplatininduced cell death by targeting the PPP2R $2 \mathrm{~A} / \mathrm{Akt} / \mathrm{mTOR}$ Axis in bladder cancer cells. J Cell Mol Med 20: 559-567, 2016.

22. Hein AL, Seshacharyulu P, Rachagani S, Sheinin YM Ouellette MM, Ponnusamy MP, Mumby MC, Batra SK and Yan Y: PR55 $\alpha$ subunit of protein phosphatase 2A supports the tumorigenic and metastatic potential of pancreatic cancer cells by sustaining hyperactive oncogenic signaling. Cancer Res 76 : 2243-2253, 2016

23. Zhang Y, Ma T, Yang S, Xia M, Xu J, An H, Yang Y and Li S: High-mobility group A1 proteins enhance the expression of the oncogenic miR-222 in lung cancer cells. Mol Cell Biochem 357: 363-371, 2011.

24. Shen S, Yue H, Li Y, Qin J, Li K, Liu Y and Wang J: Upregulation of miR-136 in human non-small cell lung cancer cells promotes Erk1/2 activation by targeting PPP2R2A. Tumour Biol 35: 631-640, 2014
25. Liu X, Sempere LF, Ouyang H, Memoli VA, Andrew AS, Luo Y, Demidenko E, Korc M, Shi W, Preis M, et al: MicroRNA-31 functions as an oncogenic microRNA in mouse and human lung cancer cells by repressing specific tumor suppressors. J Clin Invest 120: 1298-1309, 2010.

26. Liang WL, Cao J, Xu B, Yang P, Shen F, Sun Z, Li WL, Wang Q and Liu F: miR-892a regulated PPP2R2A expression and promoted cell proliferation of human colorectal cancer cells. Biomed Pharmacother 72: 119-124, 2015.

27. Wong QW, Ching AK, Chan AW, Choy KW, To KF, Lai PB and Wong N: MiR-222 overexpression confers cell migratory advantages in hepatocellular carcinoma through enhancing AKT signaling. Clin Cancer Res 16: 867-875, 2010.

This work is licensed under a Creative Commons Attribution-NonCommercial-NoDerivatives 4.0 International (CC BY-NC-ND 4.0) License. 